\title{
Predictors of adherence to a gluten-free diet in coeliac disease: do knowledge, attitudes, beliefs, quality of life and symptoms play a role?
}

\author{
Eirini Dimidi ${ }^{1}$, Bonita Amilah ${ }^{1}$, Aikaterini Ageridou ${ }^{1}$, Charlotte Foster ${ }^{1}$, Jaspreet Singh ${ }^{1}$, \\ Paul Ciclitira ${ }^{1,2}$, Patrick Dubois ${ }^{3}$ and Kevin Whelan ${ }^{1}$ \\ ${ }^{1}$ Department of Nutritional Sciences, King's College London, London, United Kingdom, \\ ${ }^{2}$ Department of Gastroenterology, Guy's and St Thomas' Hospitals NHS Trust, London, United Kingdom and \\ ${ }^{3}$ Department of Gastroenterology, King's College Hospital, London, UK.
}

Coeliac disease is a chronic autoimmune enteropathy causing small intestinal inflammation, triggered by gluten ingestion. Strict lifelong adherence to a gluten-free diet (GFD) is the only available management strategy, however adherence varies among patients with coeliac disease. Studies have investigated the association between GFD adherence and knowledge, attitudes, experiences, symptoms or quality of life (QoL). These factors are often investigated in isolation, and yet may well themselves be related, however there is limited research on the associations among these variables and with their association with adherence to a GFD. The aim of this study was to identify the relationship between adherence to a GFD and demographic characteristics, knowledge, attitudes and beliefs regarding a GFD, experiences of following a GFD, symptoms and QoL.

Patients with coeliac disease were recruited from specialist coeliac disease clinics in secondary care and completed a series of validated questionnaires. Adherence to GFD was assessed using the 'Coeliac Dietary Adherence Test' (CDAT) and 'GFD-Score' (GFD-S). Knowledge was measured using a 7-question knowledge questionnaire on gluten-containing foods and benefits of gluten exclusion. Attitudes were measured using the 'Beliefs about Medicines Questionnaire' (beliefs about the necessity of a GFD) and the 'Revised Illness Perception Questionnaire' (beliefs about coeliac disease). QoL was measured using the 'Coeliac Disease Questionnaire'. Symptoms were measured using the Coeliac Symptom Index questionnaire. Multivariate logistic regression was performed to identify associations between adherence to a GFD and demographic characteristics, beliefs, symptoms, QoL, knowledge.

Overall, 116 patients with coeliac disease were included (47 \pm 16 years, $70 \%$ female, $8.8 \pm 10.1$ years since diagnosis). Based upon the CDAT questionnaire, $58(50 \%)$ adhered to a GFD, whereas based upon GFD-S $86(74 \%)$ adhered to a GFD. Using CDAT adherence as an outcome, logistic regression revealed that being male was associated with being non-adherent to a GFD $(\mathrm{OR}=0.36, \mathrm{p}=$ 0.028). Having higher scores of emotional QoL was associated with being adherent to a GFD (OR $=1.19, \mathrm{p}<0.001)$. Using GFD-S adherence as an outcome, being a member of a patient support group $(\mathrm{OR}=3.8, \mathrm{p}=0.012)$, having strong beliefs about coeliac disease and the necessity of a GFD $(\mathrm{OR}=1.03, \mathrm{p}=0.030)$, and reporting that coeliac disease is a chronic condition $(\mathrm{OR}=1.2, \mathrm{p}=0.008)$ were associated with being adherent to a GFD. Experiencing difficulties when eating with family/friends was associated with nonadherence $(\mathrm{OR}=0.98, \mathrm{p}=0.007)$. Knowledge of the GFD was not a predictor of being adherent in the multivariate regression analysis.

The significant association between sex, attending support groups, QoL, beliefs of coeliac disease and the GFD, and experiences with adherence to a GFD needs to be considered by dietitians and gastroenterologists managing patients with coeliac disease. Recent introduction of methods to quantify gluten in both urine and faeces of affected subjects will facilitate this. Future larger studies are needed to examine whether additional education or support improve adherence to a GFD in patients with coeliac disease and the factors associated here should be the target of such interventions. 